\title{
Zoos et cause animale
}

Perspectives éthologique et anthropologique

Zoos and the Animal Cause. Ethological and Anthropological Perspectives

\section{Frédéric Joulian et Christophe Abegg}

\section{(2) OpenEdition}

Journals

Édition électronique

URL : https://journals.openedition.org/tc/3945

DOI : $10.4000 /$ tc. 3945

ISSN : 1952-420X

Éditeur

Éditions de l'EHESS

Édition imprimée

Date de publication : 31 décembre 2008

Pagination : 120-143

ISSN : 0248-6016

Référence électronique

Frédéric Joulian et Christophe Abegg, «Zoos et cause animale », Techniques \& Culture [En ligne], 50

2008, mis en ligne le 31 décembre 2010, consulté le 29 septembre 2022. URL : http://

journals.openedition.org/tc/3945; DOI : https://doi.org/10.4000/tc.3945 


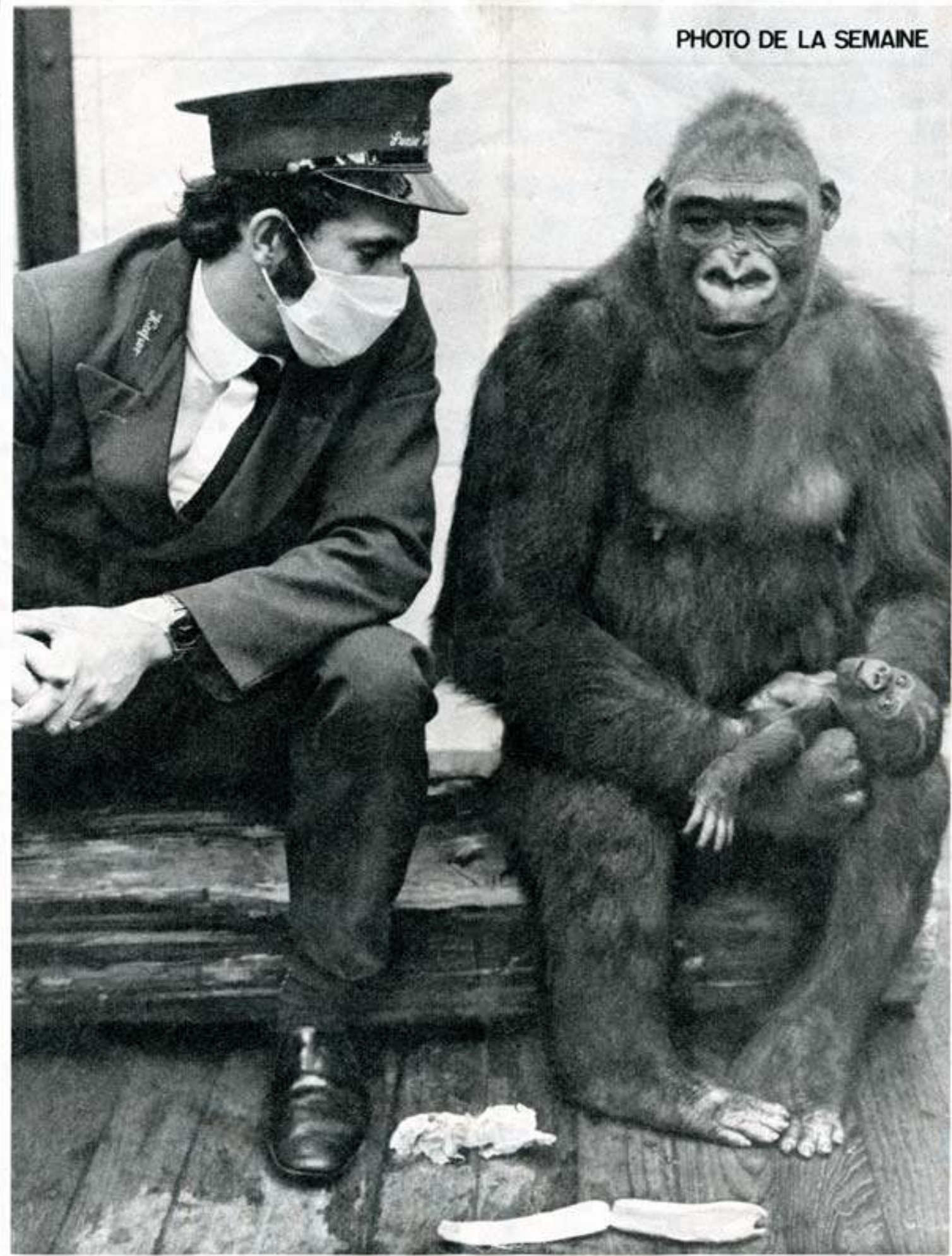

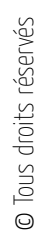




\title{
ZOOS ET CAUSE ANIMALE
}

\section{Perspectives éthologique et anthropologique}

\author{
Les auteurs ont l'un et l'autre travaillé sur des animaux encagés et en milieu \\ naturel. Ils joignent leur double expérience afin de réfléchir au statut indécis des \\ zoos contemporains en relation avec les questions plus larges de conservation \\ animale.
}

Les animaux montrés dans les parcs zoologiques sont, à l'image de ceux des grottes du Paléolithique, le produit d'un choix, d'une vision du monde, l'expression d'un imaginaire original. Même s'ils apparaissent au visiteur bien vivants, mobiles, odorants, ils constituent un bestiaire, mais un bestiaire de plus en plus controversé. Les zoos exposent des animaux sauvages, renvoient à des parts de natures lointaines et exotiques. Ils sont doubles, tout à la fois hérités, « patrimoniaux » pour certains, lieux de modernité et de spectacle pour d'autres. Pour les chercheurs actuels, qu'ils soient du côté des sciences de la nature ou des sciences de la culture, il importe de réfléchir sur les zoos en tenant compte des choix passés et de penser à nouveaux frais cette relation originale avec une nature « originelle », généralement prise comme atemporelle et prototypique. Quelle était la nature, il y a 150 ou 200 ans lors de la création des zoos d'Occident et quelle est-elle à présent? Nous ne nous livrerons pas ici à un travail d'historien, mais disons que les zoos constituent une forme particulière de cette relation changeante de l'homme à la nature dont il convient d'analyser les modes d'existence et de légitimation. 


\section{« Appauvrir »}

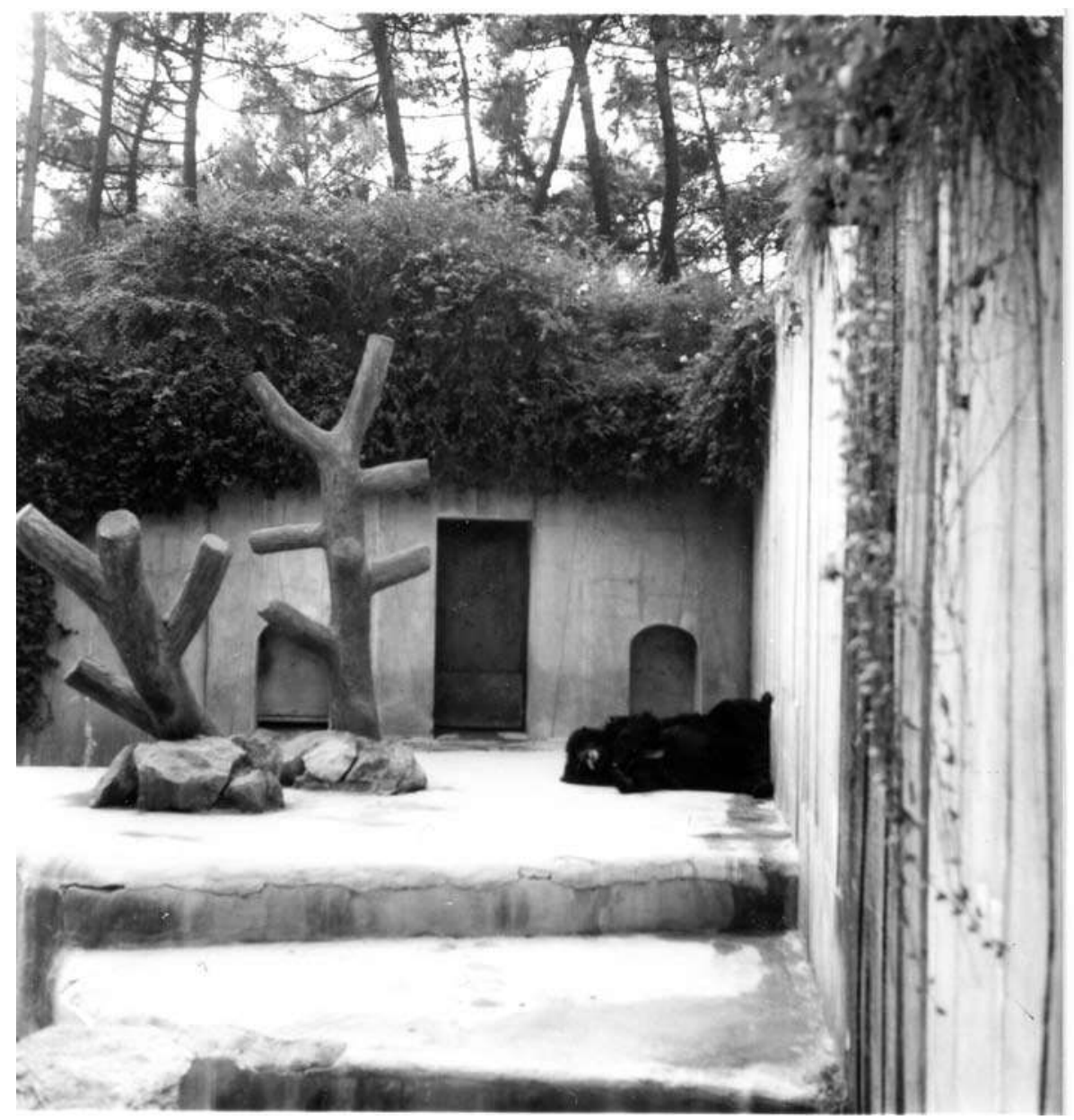

Zoo de la Palmyre, Charente-Maritime

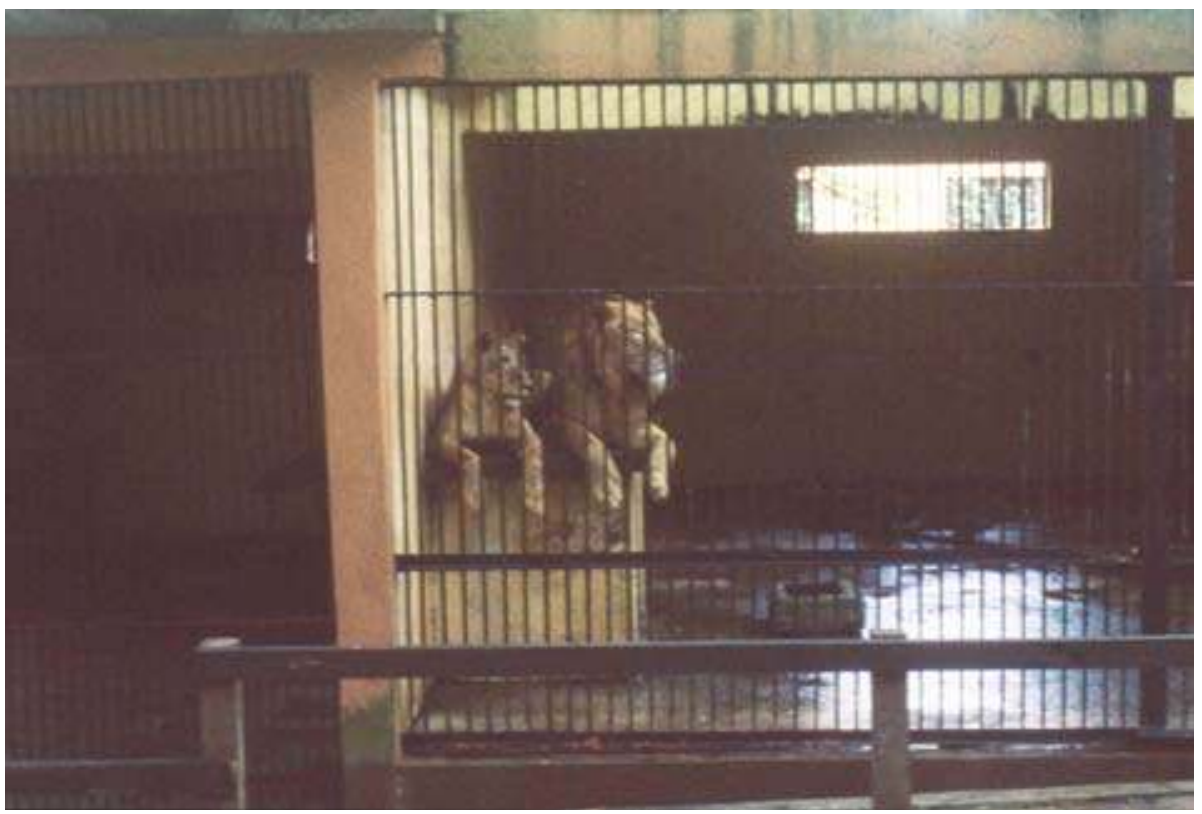


Depuis leurs origines, les zoos ont rempli diverses fonctions. Par exemple, en France, à sa création, la ménagerie du Jardin des plantes répondait à des logiques taxinomiques et zoologiques, elle participait d'un ensemble dans lequel entraient également les différentes collections du Muséum d'histoire naturelle (Brouard 1994, Baratay \& Hardouin-Fugier 1998, Mullan \& Marvin 1999). Dès le XIX ${ }^{\mathrm{e}}$ siècle, les zoos connurent un véritable succès populaire, ce qui en fit des lieux d'attraction et de promenade en famille. Durant l'époque d'expansion coloniale, les pays européens exhibèrent fièrement, comme des trophées vivants, les animaux exotiques rapportés de pays lointains. Les hommes furent même montrés dans des dispositifs d'expositions analogues et cela à l'époque de création du Parc de Vincennes en 1931 (Bancel 2004).

Au cours des deux dernières décennies, la situation a radicalement changé et les contradictions ont éclaté entre les attentes d'un public souhaitant voir des animaux sauvages et les modes de contention rustiques hérités d'une autre époque. Pourtant, les animaux nés en captivité ne peuvent être ni relâchés dans leur milieu d'origine, sans d'immenses difficultés liées aux impacts sur les communautés animales et humaines voisines ou aux faibles chances de survie de l'animal, ni supprimés pour des raisons éthiques. Dans de telles conditions, quelles sont les raisons généralement invoquées pour le maintien d'animaux sauvages en captivité? Les trois principaux arguments avancés par les zoos sont les suivants: la conservation d'espèces rares, la recherche scientifique, l'éducation informelle du public. Nous les mettrons par la suite en relation avec les savoirs éthologiques et anthropologiques qui permettent de les contextualiser et de les évaluer.

Nous laissons en revanche à la sociologie de la culture ou des publics le soin d'aborder la question des usages et de l'économie de ces établissements. Elle est bien évidemment essentielle à une bonne compréhension des changements actuels (voir Chaumier ce numéro).

\section{Le zoo, acteur écologiste?}

La montée de préoccupations écologiques et d'une éthique animale, depuis une vingtaine d'années, a favorisé l'émergence d'un sentiment négatif envers les zoos dans certains secteurs de l'opinion publique. Les mouvements de lutte contre l'expérimentation sur l'animal, l'engagement de la fondation Brigitte Bardot contre certains modes d'abattage, celui des top models refusant de défiler avec des fourrures, les militants de Greenpeace s'interposant physiquement aux baleiniers, les pétitions dénonçant le massacre des éléphants entretenu par le commerce international de l'ivoire témoignent d'une sensibilité accrue de l'opinion face à la souffrance animale (Jeangène Vilmer 2008). Les détracteurs des zoos se sont faits plus nombreux: la vision d'un animal restreint dans ses mouvements et maintenu dans un volume réduit et bétonné a suscité une 


\section{« Appauvrir »}

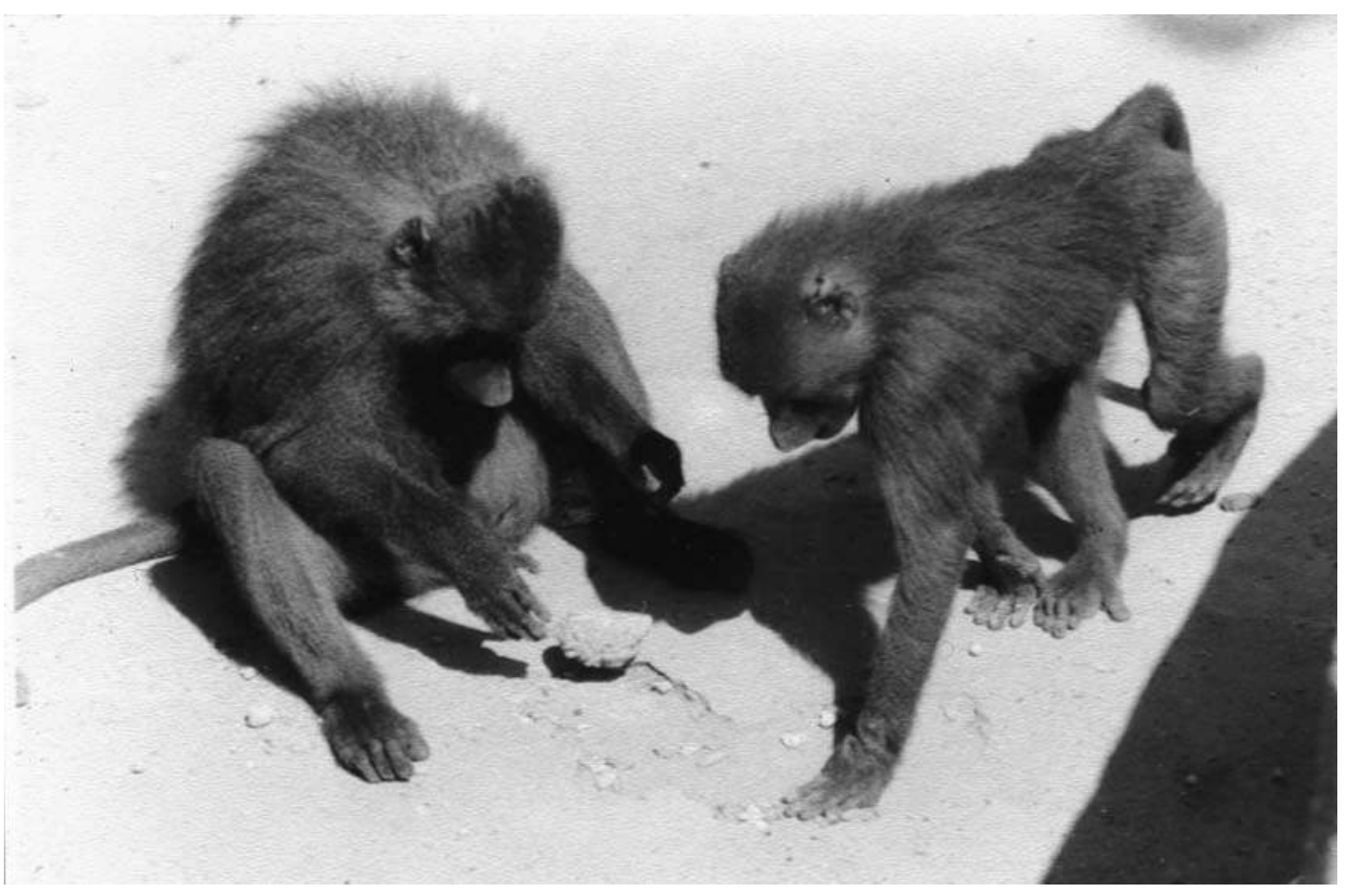

\section{Babouins}

Les babouins du zoo de Vincennes ont développé une innovation technique originale d'utilisation d'outils en pierre avec lesquels ils cassaient le substratum de leur enclos et allaient fouir. Les responsables du zoo, peu de temps après, leur ont enlevé leurs instruments et ont cimenté l'enclos (Abegg \& Thierry 1998), photos C. Abegg.

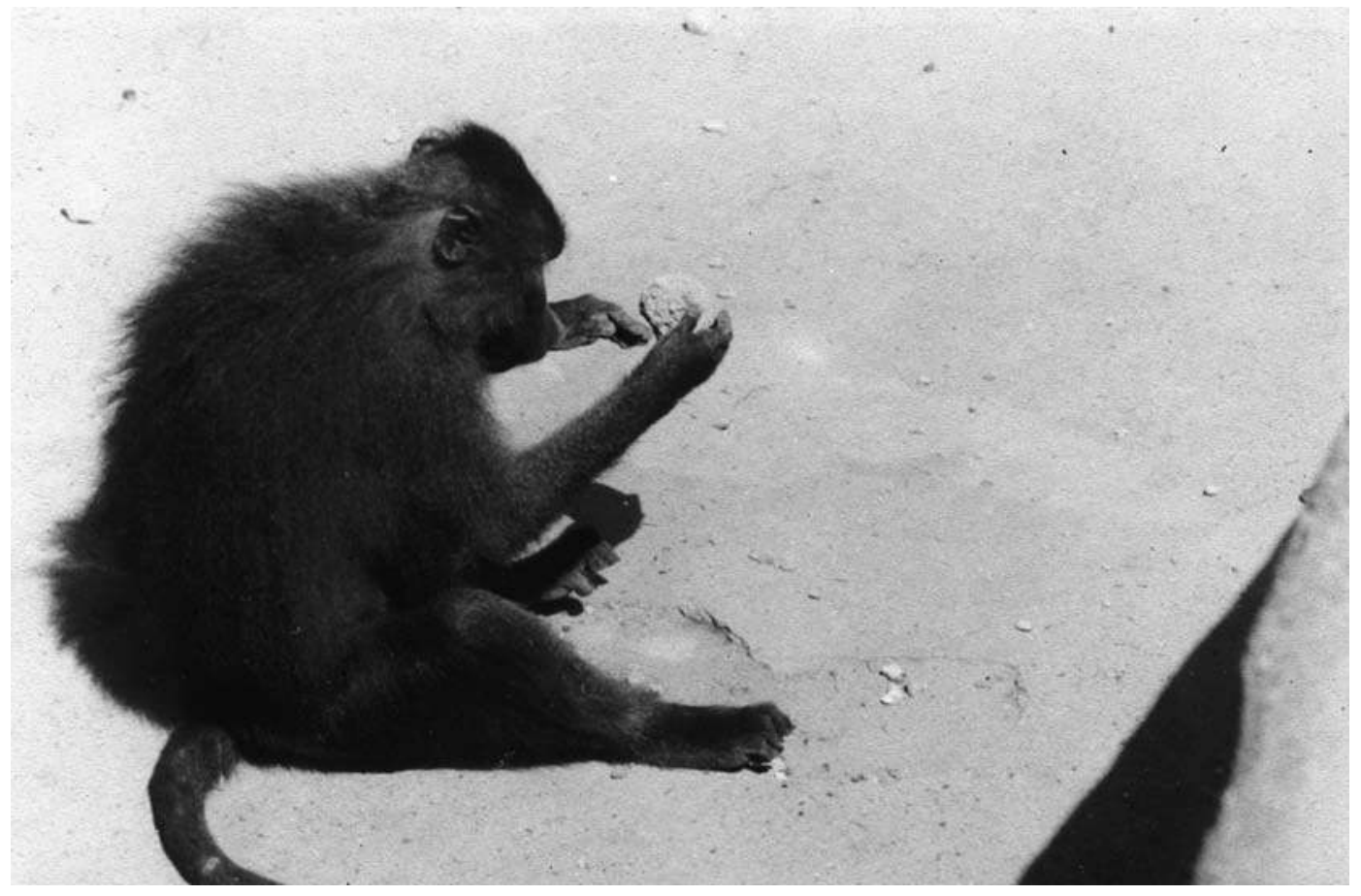


compassion de plus en plus marquée et la désaffection d'un grand nombre de jardins zoologiques. Ces derniers ont dû fermer leurs portes ou revoir leur politique de gestion animale et/ou de communication.

Face à la baisse de fréquentation, les responsables des parcs zoologiques ne pouvaient rester inactifs. Ce rejet des zoos en tant que structures " carcérales » a poussé les responsables de ces institutions à changer de méthodes et de discours. Reprenant le credo écologiste à leur compte, ils ont mis en relief la destruction inexorable des richesses naturelles de la planète pour justifier l'existence des zoos comme conservatoires d'espèces en voie de disparition. Un discours plus ouvert, dépassant le simple cadre de la fonction de divertissement, s'est donc progressivement élaboré, mettant en avant une mission de conservation et de reproduction d'espèces menacées. Des programmes internationaux de gestion rationnelle des populations animales captives ont été mis en place. Ils permettent de gérer la reproduction de chaque espèce menacée à l'échelle de la population mondiale captive en s'assurant du maintien de la diversité génétique par des échanges entre zoos (EAZA). De plus, ces programmes permettent une concertation renforcée dans la résolution des problèmes techniques de reproduction et dans la définition des recherches nécessaires. Une telle coordination des efforts de gestion ouvre-t-elle pour autant la voie à des réintroductions d'espèces ayant disparu de leur milieu naturel? Vingt années de réintroductions pilotées ou non par les zoos relativisent très fortement de telles démarches.

À cette proposition de conservation développée par les zoos s'ajoutent des sauvetages d'urgence de quelques espèces rares. Un parc zoologique devient un laboratoire ayant aussi pour objectif de conserver des espèces précieuses et d'étudier prioritairement la physiologie de leur reproduction. Suivant cette logique, il s'agirait pour certains de parer au plus pressé en sauvant ce qui est sur le point de s'éteindre. Mais cette « arche de Noé » portée par les zoos a-t-elle des effets tangibles et durables en termes d'impacts sur les populations animales et milieux naturels? On peut sérieusement en douter.

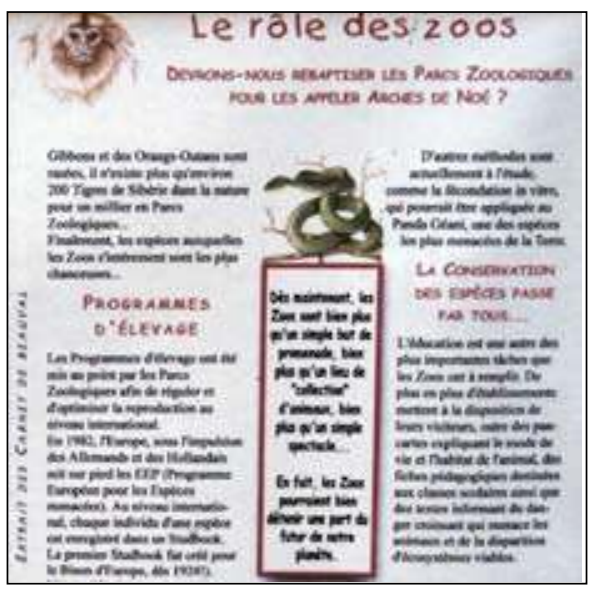


Il est vrai que cette logique de conservation a initialement été appuyée par des exemples de réintroductions réussies. Pour n'en citer qu'un des plus connus, le bison d'Europe disparaît de France au VII siècle et le dernier bison libre s'est éteint en Pologne en 1919. Les 54 individus existant encore dans des zoos en 1923 ont permis cependant de sauver l'espèce et de la réintroduire dans la réserve forestière polonaise de Bialowieza. Les réintroductions rencontrent toutefois souvent des obstacles. Ainsi, le tamarin lion, petit singe disparu des forêts côtières fortement dégradées du Brésil, n’a pu être réacclimaté qu'après des efforts excessivement coûteux par rapport aux résultats obtenus. Il a fallu une dizaine d'années et de multiples tentatives infructueuses avant que l'on ne corrige les facteurs d'inadaptation de quelques sujets captifs aux conditions de la vie en forêt tropicale. La réintroduction du lynx dans les Vosges, elle, s'est heurtée à l'opposition des chasseurs et des éleveurs. En 1992, le naturaliste indien Karanth (1992) a critiqué le projet d'introduction des macaques queue de lion pour renforcer les populations existantes du Sud de l'Inde dans un habitat aujourd'hui fragmenté de forêts tropicales, de plaines et de montagnes. La mise en ouvre d'un seul projet pour introduire 12 macaques captifs, même bien planifié et exécuté, coûtait 150000 dollars! En revanche, la protection efficace d'une forêt dense de $250 \mathrm{~km}^{2}$ contenant de 250 à 500 macaques sauvages, nécessitant l'appui de 50 gardes supplémentaires, ne coûtait que 30000 dollars par an. En outre, selon Karanth, si l'on introduit des macaques dans des forêts où leurs congénères sauvages subissent déjà une pression due à la chasse et à la détérioration de leur habitat, les nouveaux arrivants seront logiquement éliminés par les mêmes causes.

Les divers exemples de réintroductions de chimpanzés dans des environnements sauvages se sont déroulés dans des conditions exceptionnelles, souvent des isolats dépourvus d'individus sauvages. Ces actions ont été réalisées sur de petites îles isolées en Gambie (Brewer 2008), au Liberia ou en Côte d'Ivoire, et ensuite dans des zones protégées et contrôlées (Help Congo... cf. Gossens et al. 2001) ou des « sanctuaires » - Lola Ya Bonobo (André 2007), Nyaru Mentengl, etc. -, sites qui se voudraient des antichambres de futurs relâchés. La question des relations avec les hommes vivant à proximité a cependant été traitée en amateur, comme secondaire, ou pilotée à des niveaux où les acteurs concernés ne pouvaient participer. Ces réintroductions « modèles » relèvent parfois d'artefacts en terre étrangère dont la pérennité (liée à l'engagement de certaines personnes ou de certaines ONG) est loin d'être assurée. En ce qui concerne les animaux relâchés au contact des groupes sauvages, ils ont fréquemment été tués par leurs congénères naturels ou par des prédateurs (comme à Azagny en Côte d'Ivoire par exemple). En fait, il est très intéressant de voir qu'un grand nombre de ces échecs ont eu lieu à une période où les animaux, fussent-ils des primates supérieurs, étaient encore gérés comme s'ils étaient dénués de compétences sociales ou de biographies originales (life histories) et où le milieu naturel était censé jouer un rôle clef en tant que révélateur de leurs capacités intrinsèques. Dans les années 1970-1980, 
les facteurs sociaux étaient encore mal évalués. Dans les années 19902000, de nouveaux projets, plus organisés, visaient « à réapprendre la vie sauvage » à des animaux « orphelins ». Ils se poursuivent aujourd'hui, plus nombreux que jamais. Ces actions attirèrent plus largement les médias et les financements que les enjeux plus globaux de gestion des animaux et des espaces naturels. Leurs vertus pédagogiques semblent accrues mais leurs ambitions de « retour à la nature » restent aussi inefficaces que les précédentes tentatives. Le problème est bien évidemment mal posé.

Plus concrètement, ces exemples soulignent les écueils pratiques et logistiques qui accompagnent les réintroductions. D'une part, les animaux captifs n'ont souvent pas l'expérience indispensable à la survie dans le milieu naturel, et d'autre part, les pressions socio-économiques ayant contribué à la disparition d'une espèce dans une zone sauvage peuvent être toujours à l'œuvre lors de la réintroduction. Largument économique est également important : il peut être plus judicieux de s'engager sur place en devançant les disparitions futures par des programmes moins coûteux de préservation que par des réintroductions. Il y a vingt ans, Edward Wilson (1989), annonçait que 4000 à 6000 espèces animales ou végétales disparaissent chaque année à cause du seul défrichement des forêts tropicales humides. Cela représente un rythme d'extinction 10000 fois plus rapide qu'avant l'apparition de l'être humain. Aujourd'hui, avec le réchauffement climatique, la surexploitation des milieux naturels, mais aussi les crises politiques génératrices d'instabilité, la disparition d'espèces animales est devenue un enjeu scientifico-politique majeur (Joulian 2004).

\section{Réorienter les recherches}

Qu'en est-il de l'argument selon lequel la recherche zootechnique justifie amplement l'existence des zoos? Nous assistons au développement de techniques modernes de reproduction des animaux comme le prélèvement et le transfert d'embryons, la cryopréservation de sperme ou l'insémination artificielle, la fertilisation in vitro ainsi qu'une meilleure connaissance des cycles hormonaux permettant de prédire les périodes de fertilité des femelles. S'agissant de problèmes aussi aigus que la disparition d'espèces, il semble légitime aux gestionnaires de parcs animaliers de mettre en œuvre ces innovations en matière de procréation artificielle. La mise en place de telles techniques de pointe coûte cependant très cher et tout investissement financier se doit d'être décidé en fonction de priorités solidement argumentées. Or, même s'il est valorisant de maîtriser ces techniques, on peut s'interroger sur la pertinence de faire se reproduire quelques espèces, souvent emblématiques (bonobo, gorille des montagnes, tamarin lion, tigre, okapi, rhinocéros, etc.), et sur leur rôle tangible dans le futur repeuplement d'écosystèmes détériorés. Que les zoos mettent au premier plan la mission de faire croître et multiplier quelques espèces rares déracinées de leur milieu naturel paraît compréhensible. En revanche, 

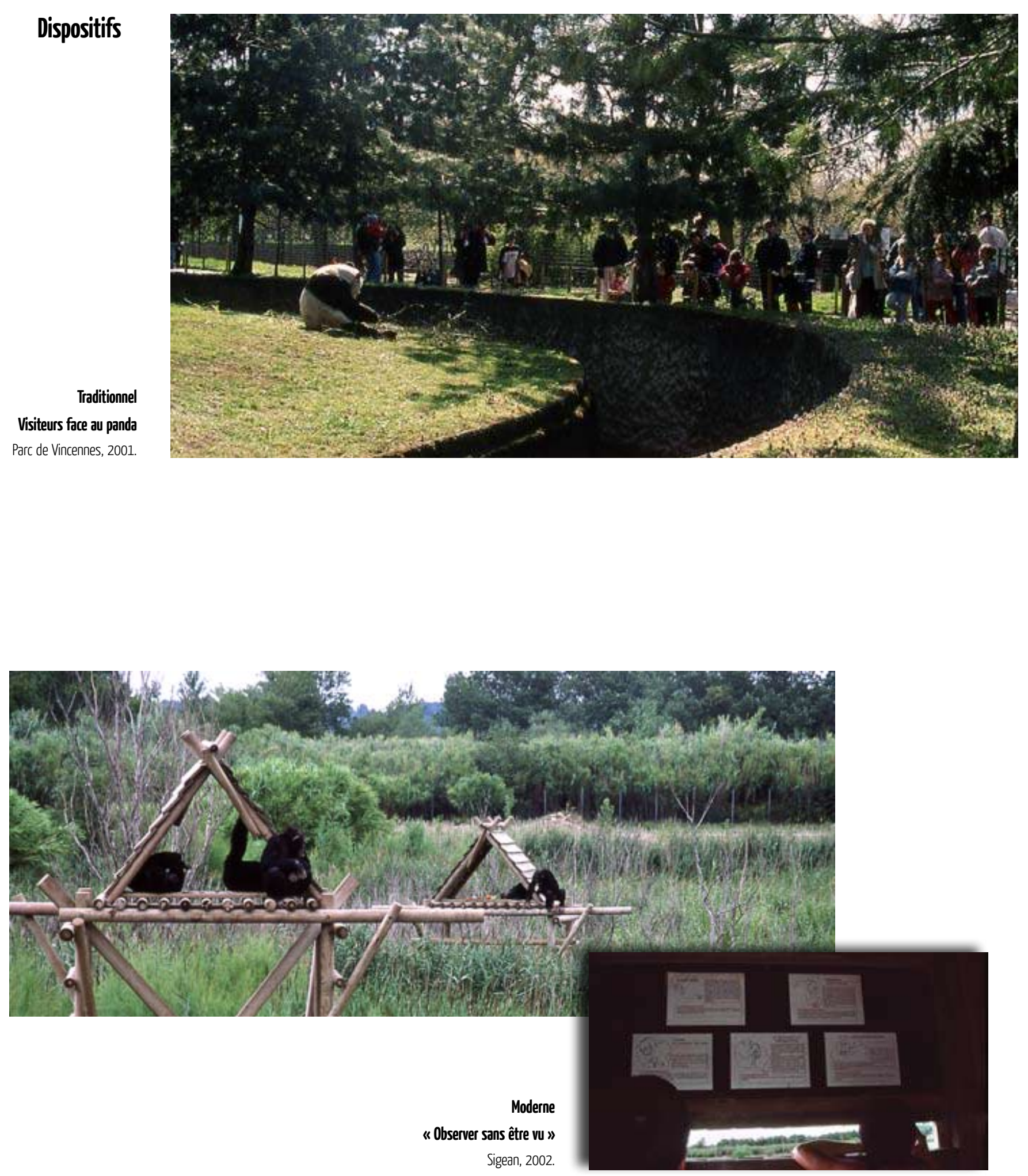

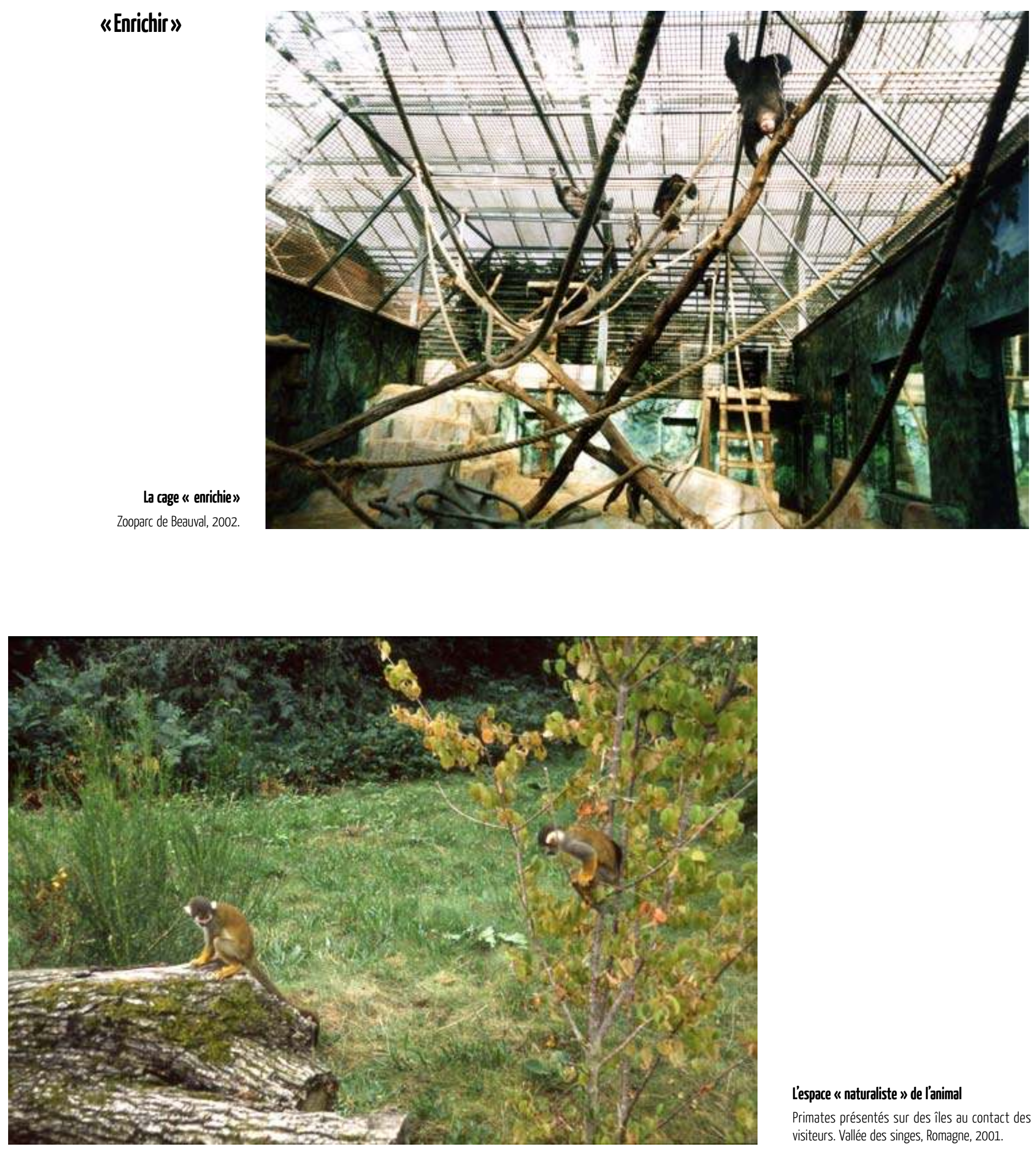

L'espace « naturaliste » de l'animal

Primates présentés sur des îles au contact des visiteurs. Vallée des singes, Romagne, 2001. 
cette perspective devient peu crédible si rien n'est fait simultanément pour lutter contre les causes de dégradation des milieux abritant les espèces menacées. Les actions de conservation et de réintroduction pilotées par des logiques biologiques et spécifiques (et elles sont très nombreuses) ont peu de chances d'aboutir et le concept « d'espèce parapluie » répond rarement aux catégories et cosmogonies locales.

Les études sur la physiologie de la reproduction d'espèces rares associées à une logique d'élevage dans un but de réintroduction sont mises en avant par certains zoos. La recherche sur le bien-être des animaux captifs, ou « enrichissement comportemental », représente une autre voie en plein essor aux États-Unis, en Hollande ou en Allemagne. Elle débute dans certains parcs français, mais elle est bien souvent tâtonnante ou impossible dans de vieilles structures ou de petits parcs animaliers. Lors de la création des premiers zoos en Europe au siècle dernier, les animaux étaient présentés seuls ou en couple dans des cages exiguës. Au début du $\mathrm{XX}^{\mathrm{e}}$ siècle, un souci de présentation plus réaliste des animaux se fit sentir, à l'exemple du zoo de Hambourg, pionnier en la matière, où des rochers artificiels furent construits. Des séparations plus discrètes, à l'aide de fossés, furent installées entre les animaux et les visiteurs. Nous savons maintenant que cette évolution, dont on voit l'héritage dans de nombreux zoos européens - comme celui de Vincennes en France -, ne témoignait pas d'une meilleure compréhension, ni d'un plus grand respect des besoins vitaux des animaux. L'univers physique et social des animaux captifs restait extrêmement pauvre et nombre d'animaux manifestaient des comportements anormaux (Joulian 2002a).

Les zoos les plus attentifs au bien-être animal présentent maintenant les animaux dans des cadres de vie mieux définis et enrichis de diverses structures adaptées aux espèces, avec le souci de respecter les besoins physiologiques, psychologiques et sociaux propres à chaque espèce. Cette nouvelle approche met l'accent sur l'importance d'environnements dits « naturalistes », c'est-à-dire inspirés autant que possible des conditions rencontrées dans le milieu naturel en opposition aux zoos qui isolent l'animal de son contexte. Il n'en reste pas moins qu'il est extrêmement difficile, voire impossible, de parvenir à recréer les conditions de l'habitat d'origine, ou plus généralement, « d'imiter la nature ». En ce sens, nous pourrions dire que l'éthologie mise en ouvre dans de telles démarches est encore dans bien des cas une éthologie simpliste. Étant donné les avancées des connaissances en matière de plasticité comportementale et d'adaptabilité de certaines espèces (des macaques ou des chimpanzés notamment pour rester dans le registre des primates), cette option mimétique relève d'une utopie surannée. La « re-création » de la nature renvoie pour certains à des conceptions naïves, pour d'autres, mais parfois les mêmes, à l'opportunité de construire un discours visant à légitimer certaines ambitions commerciales ou idéologiques.

Ces zoos « naturalistes » introduisent aussi l'idée d'enrichissement des conditions de vie de l'animal, idée qui a mis très longtemps à s'imposer dans les établissements français, et plus généralement, latins. Elle implique 


\section{«Hors du Z00»}

Animal de ferme, en estive

Ariège, 2006.

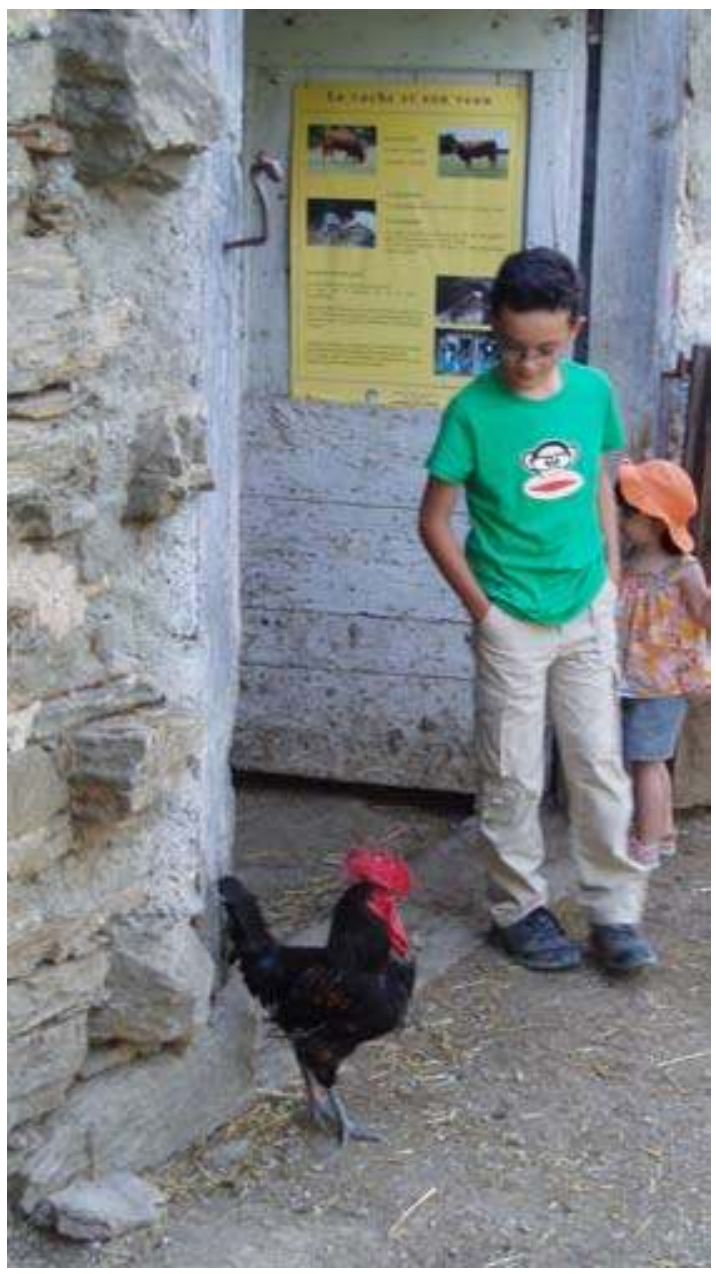

Animal de ferme, à l’Écomusée

Ariège, 2007. 
une rupture conceptuelle dans la façon de penser l'animal, non plus comme passif et interchangeable, mais comme un être avec un corps et un esprit agissants. En 1925 déjà, le primatologue américain Yerkes résumait son expérience de l'élevage des grands singes en soulignant l'importance du « travail » (au sens où les animaux ont à fournir un effort dans l'exploration de leur milieu) parmi les qualités d'un bon environnement de captivité. Malgré ces conseils formulés il y a plus de quatre-vingts ans, beaucoup de professionnels de zoos pensent encore qu'il est normal de satisfaire aux besoins des animaux sans qu'ils aient d'efforts ou de « travail » à fournir. Ce statut « d'invités privilégiés » permettrait-il ainsi de mieux distinguer les animaux sauvages (du zoo) des animaux domestiques et de travail? Pour comprendre ces représentations contrastées mais ténues, il faudrait analyser plus largement les débats, les changements, les ruptures d'idées et de pratiques tout au long du siècle passé mais aussi le nouveau statut des animaux de travail, leur remplacement progressif par les machines dans l'agriculture et le transport et bien sûr le développement considérable des animaux de compagnie.

\section{Yinti, animal de compagnie,}

Marseille, 2007

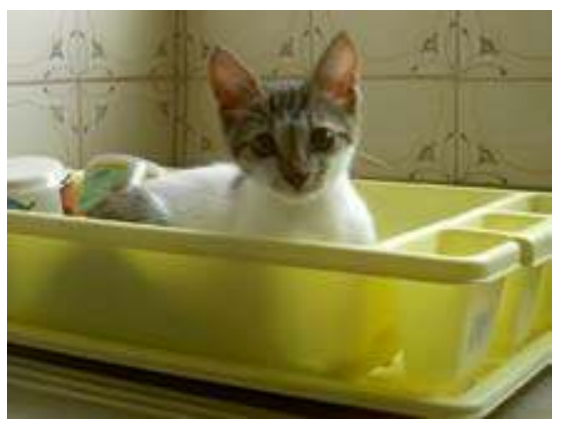

Mais revenons aux sujets des zoos; si nous prenons la peine d'envisager le temps et les compétences consacrés par un primate en milieu naturel dans sa recherche d'aliments, il est facile de saisir qu'il manque à l'animal captif l'un de ses principaux motifs d'existence. Plus d'une centaine de plantes et des dizaines de petits animaux composent souvent son régime alimentaire dans la nature; il faut qu'il puisse les repérer dans un écosystème complexe, tout en évitant de s'empoisonner ou de se retrouver nez à nez avec un prédateur. Cette recherche occupe généralement les deux tiers de son temps en milieu naturel. La quête de nourriture est une activité parfois solitaire mais souvent collective et socialisante; elle fait intervenir des mécanismes d'apprentissage individuels et sociaux, et les jeunes acquièrent au sein du groupe une expérience indispensable à la vie adulte.

Par contraste, il est extrêmement frappant d'observer l'ennui extrême d'un primate captif. Un sol en béton dans un espace simplifié est certes plus facile à fabriquer et à nettoyer, mais les conséquences sont désastreuses pour les animaux. Le manque de possibilités d'exploration vide le quotidien de ses saillances, de son sens et de son intérêt. Les conditions appauvries des zoos génèrent fréquemment des comportements comme la coprophagie, 
des automutilations, des balancements et des va-et-vient stéréotypés, voire des infanticides. À l'inverse, le simple fait qu'un animal ait à explorer son environnement pour trouver sa nourriture améliore notablement son bienêtre et diminue la fréquence des comportements pathologiques.

Ce n'est qu'assez récemment que des progrès ont été accomplis à partir de la compréhension des besoins spécifiques des animaux captifs. Cette approche «naturaliste » s'appuie sur des recherches éthologiques et environnementales aussi bien sur le terrain qu'en captivité et vise à mettre au point des environnements « semi-naturels » (au passage, il faudrait s'arrêter sur ce lexique imprécis : « semi-captivité », « semi-liberté », etc., qui illustre bien les contradictions de la science à propos de la question animale). Les espaces, très souvent agrandis, sont agrémentés de végétation plantée, de rochers, de cours d'eau, de structures en bois modifiables par les animaux, et parfois, plusieurs espèces sont placées ensemble avec succès. De tels environnements présentent un double avantage: d'une part, ils mettent les animaux dans des situations plus proches de celles qu'ils rencontrent dans la nature, d'autre part, ils permettent de présenter au public des animaux ayant des comportements plus cohérents avec l'éthogramme de l'espèce en milieu naturel. Ainsi, au zoo d'Arnhem en Hollande, une colonie d'une trentaine de chimpanzés a été présentée de façon optimale pour l'observation des comportements par le public ou les chercheurs. Le groupe approche par sa taille et sa composition ceux rencontrés dans la nature. L'espace de plus d'un hectare est couvert d'herbe, de sable, et d'une cinquantaine d'arbres (et protégé cependant par des barrières). Le primatologue Franz De Waal a pu y réaliser une étude publiée en 1982 et mondialement connue: Chimpanzee Politics, Power and Sex among Apes. Laccent mis sur les conditions améliorées de captivité augmenterait donc le bien-être des animaux et accroîtrait en retour la valeur des recherches sur le comportement. À l'inverse, remarquons que l'élimination violente de Luit par une coalition de mâles rivaux durant une nuit (Waal 1986) choqua vétérinaires et éthologues et ne fut guère discutée, ni auprès du public, ni dans la communauté scientifique.

Le rapport entre recherches en captivité et en milieu naturel s'est ainsi enrichi vers la fin des années 1980. Nous rappellerons, à ce propos, le fait que le comportement de réconciliation (peacemaking) a d'abord été compris en captivité, à Arnhem, avant d'être reconnu en milieu naturel, comme un invariant comportemental des chimpanzés communs (Waal 1992). Des études sur les conditions de captivité permettent également de mieux réussir d'éventuelles réintroductions. Nous avons signalé plus haut que la réintroduction de tamarins lions dans leur habitat d'origine au Brésil fut tout d'abord un échec. Les petits singes, une fois relâchés, n'ont pu survivre car ils ne savaient ni échapper à leurs prédateurs naturels en utilisant le bout des branches, ni trouver eux-mêmes leur nourriture. Les chercheurs ont alors changé les conditions de captivité avant la libération dans le milieu naturel: ils placèrent dans les cages des supports mobiles et souples à la place des éléments rigides et ils y cachèrent la nourriture pour imiter les situations naturelles. Une fois relâchés les singes survécurent. 
Par ailleurs, même si les chances de succès à la réintroduction d'animaux captifs sont faibles, cet objectif peut également servir de moyen d'évaluation de la qualité d'un environnement de captivité.

Outre les avantages concernant le bien-être des animaux, la qualité des contextes d'observations ou les réintroductions, les environnements naturalistes donnent aussi de bons résultats en matière de reproduction. Avant de se poser la question d'une déficience biologique, il vaut mieux vérifier si un problème d'élevage constaté chez une espèce particulière n'est pas lié à de mauvaises conditions de captivité. Très souvent, lorsqu'un petit mammifère vient au monde et que la mère ne peut l'élever ellemême, le personnel choisit de l'élever au biberon, particulièrement s'il s'agit d'une espèce rare pour laquelle l'objectif de rendement est au centre des préoccupations des gestionnaires du zoo. Beck et Powers (1988) ont pourtant montré, pour des gorilles de plaine captifs, que $71 \%$ des femelles élevées par leur mère s'occupent de leurs petits alors que seulement $35 \%$ de celles élevées par l'homme développent une telle attitude. De plus, les femelles élevées par leur mère donnent naissance à deux ou trois fois plus de petits que ne le font celles élevées par leurs gardiens. Des observations similaires ont été réalisées chez le macaque rhésus et chez le chimpanzé. Chez beaucoup de mammifères, il n'existe pas de connaissance innée concernant le soin à apporter aux petits. Un environnement naturaliste permet donc d'améliorer le succès de la reproduction (en termes de soins apportés aux jeunes par les parents), tout autant que de restituer l'expression des comportements sociaux propres à chaque espèce. Il n'en demeure pas moins qu'il génère des modifications comportementales substantielles mêmes si non pathologiques: par exemple chez les chimpanzés, le changement de comportement de dominance des femelles dans des groupes où les mâles sont sous-représentés.

L'élevage d'espèces en voie de disparition montre aussi ses limites en tant qu'argument central de la fonction du zoo, car il tend à accréditer l'idée que l'on pourra sauvegarder la faune sauvage en la recréant artificiellement dans des îlots de nature. Cette option permet peut-être de pouvoir « sauver » une espèce in extremis ou d'attirer l'attention sur le patrimoine naturel en danger, mais ne répond pas de façon réaliste aux problèmes concrets de protection tels qu'ils se posent dans les milieux d'origine. Là où des zones sauvages existent encore, les dégradations subies par l'environnement sont généralement liées à des causes économiques, culturelles, ou politiques. Les environnements « intacts » apparaissent rapidement comme des fictions dès lors que l'on travaille en historien ou en ethnologue et que l'on se plonge dans le passé, fût-il récent (Chouin 2002, Leblan \& Joulian 2006, Leblan 2007 pour l'Afrique de l'Ouest). Qu'il s'agisse d'ours des Pyrénées, de gorilles du Rwanda, de babouins commensaux de l'Arli au Burkina-Faso ou de macaques d'Arashiyama au Japon, il convient, si l'on désire comprendre toutes les composantes des relations hommes/animaux, de se pencher sur les conceptions et pratiques locales, et ce, que les hommes habitent à proximité où dans des zones désignées par des décisions de protection. Les problèmes complexes de 
préservation sortent la plupart du temps du champ d'entendement et d'action des zoos, mais aussi de bon nombre d'institutions internationales ou d'ONG, et ne sont traités aujourd'hui que de façon chirurgicale au regard des logiques contradictoires qui les traversent. Les grands organismes (PNUE, WWF, IUCN, etc.) préfèrent des formules globales et aisément communicables (sauvegarder une espèce emblème, piéger le carbone) aux approches complexes des sciences sociales. Ces agendas globaux s'accordent aux discours moralisateurs et catastrophistes en vogue, (Muséum 2007, Pétition, 2008). La réalité des milieux naturels et humains est cependant plus variée, plus complexe, et donc plus difficile à transmettre et administrer. À titre d'exemple, John Oates (2006) montre que dans les espaces naturels d'Afrique de l'ouest, un grand nombre de régions sont encore très faiblement investies par la recherche et que les propos alarmistes sur la disparition des anthropoïdes (Walsh et al. 2003) ne peuvent être extrapolés à l'ensemble de la sous-région et encore moins à toute l'aire de distribution. Nos propres travaux en Côte d'Ivoire en 1999 et 2000 montrent, contre la doxa zoologique à l'œuvre (Boesch \& Tomasello 1998, Whiten et al. 1999), l'existence de chimpanzés aux portes d'Abidjan (Joulian 1995, Jankowski 2000) ou de populations vivant dans les zones périphériques du parc de la Comoé, à proximité d'un village de chasseurs (mais absentes de ce plus grand parc naturel d'Afrique de l'Ouest, Joulian 2002b). Nos recherches en 2007 au Ghana montrent la présence de chimpanzés dans des zones dont ils sont déclarés absents depuis plus de 50 ans (Joulian et al. 2007)! De telles découvertes ont été rendues possibles en allant à contre-courant de ce que les bailleurs de fonds et le public attendent en termes de « sauvetage urgent d'animaux sauvages » et de distribution géographique des populations. Mais aussi, en conciliant de façon articulée, complémentaire et équitable les démarches de l'éthologie naturaliste et de l'anthropologie sociale ${ }^{2}$. La conservation utilise de plus en plus les ressources de la communication de masse qui vont de pair avec la défense de "causes animales », causes simples et bonnes. Sont-elles justes pour autant? Peu importe, elles n'ont généralement, faut-il le rappeler, aucun sens en termes scientifiques.

\section{Observer, comprendre et respecter}

Dans l'article 10 de sa déclaration de 1989, la Ligue internationale pour les droits de l'animal propose: " l'éducation et l'instruction publiques doivent conduire l'homme, dès son enfance, à observer, à comprendre et à respecter les animaux ». Se fondant sur de telles propositions, les zoos pourraient bien être des lieux propices à la diffusion de connaissances éthologiques et anthropologiques. Notons au passage le défaut patent du système éducatif français et des manuels du secondaire qui ne donnent guère de clefs de lecture du comportement animal aux écoliers, ni encore moins de connaissances sur les sociétés humaines, mais 


\section{Écologie vs économie?}
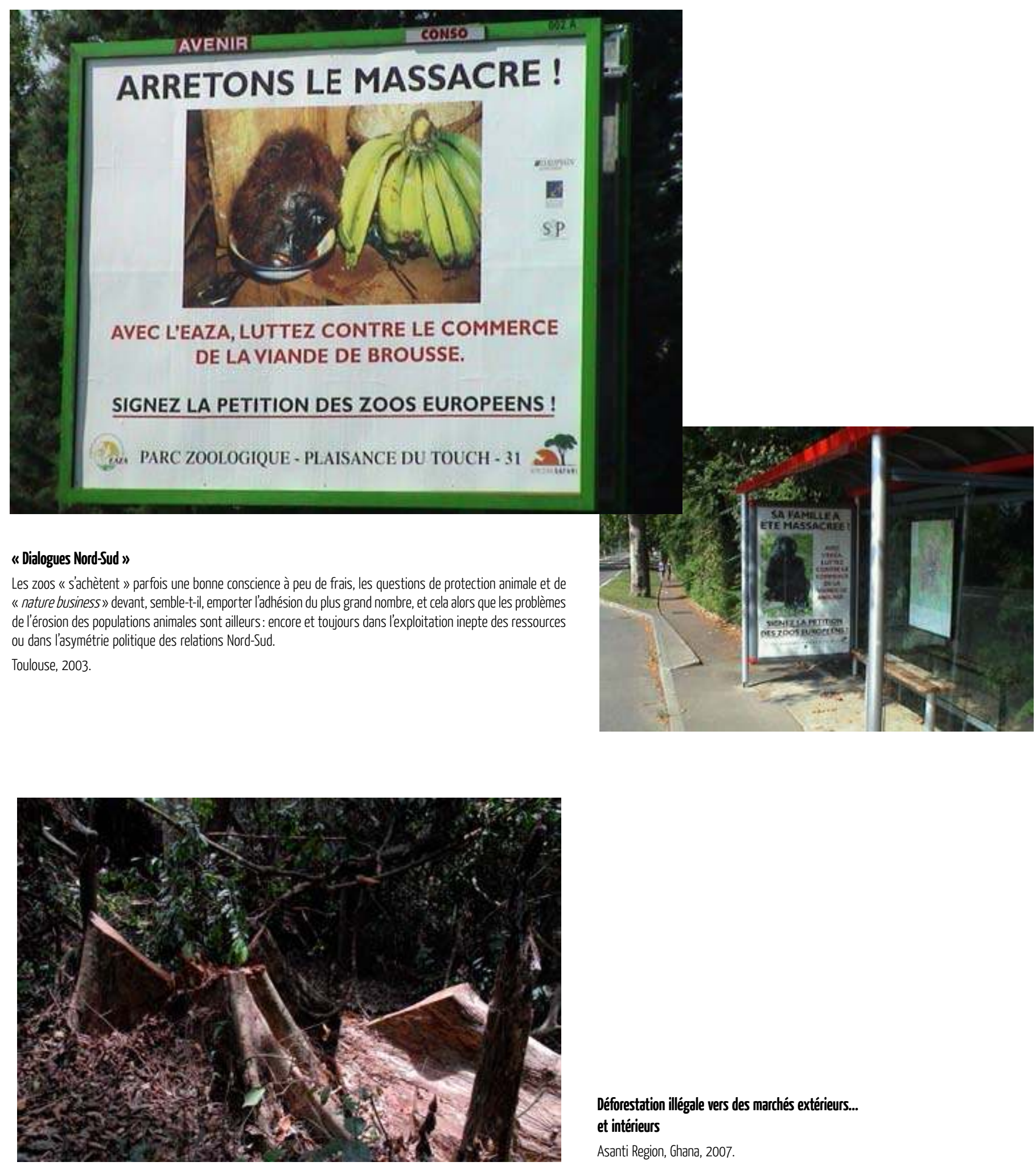

Déforestation illégale vers des marchés extérieurs... et intérieurs

Asanti Region, Ghana, 2007. 
également celui des institutions muséales plus promptes à catégoriser les espèces qu'a expliciter leurs agissements dans des cadres incitant aux respects des uns et des autres.

D'autres options, « de toucher » plus que « de voir », sont toutefois mises en place par les zoos qui donnent la possibilité aux enfants d'interagir avec des animaux domestiques, présentés dans des fermes à l'intérieur des zoos. Le contact direct devient possible et peut même être encouragé. Cette nature domestique en modèle réduit est pourtant tout aussi éloignée de la réalité - par rapport à une exploitation agricole - que la nature sauvage. Souvent conçue comme un espace récréatif pour les plus petits, elle s'accorde bien avec les données sur les motivations des visiteurs. La dimension récréative des zoos s'est d'ailleurs développée au cours de la décennie passée et les animaux ne sont plus seulement présentés pour ce qu'ils sont mais pour ce qu'ils font. Cette nouvelle donne performative s'exprime désormais dans toutes sortes d'établissements avec des rapaces, des otaries, des dauphins, etc., dans le cadre de mises en spectacles. Les frontières du zoo, du parc d'attraction, du spectacle historique ou du cirque deviennent de plus en plus compliquées. L'animal présenté au zoo n'est plus seulement le représentant de son espèce, le taxon ou le « porteparole », il devient parfois aussi animal de « show » amené à démontrer ses capacités ou à impressionner, comme au cirque.

Si nous quittons ces espaces théâtraux et revenons aux espaces réels que sont censés représenter les zoos, la prise de conscience de la fragilité extrême de certains écosystèmes s'étend de jour en jour. Dans les années à venir, la tâche de bon nombre d'acteurs, scientifiques ou pas, est de contraindre ce processus en agissant directement là où ces espaces naturels se réduisent de façon critique. Laction, pour être efficace, devra porter sur les régions qui comprennent des superficies suffisamment importantes et riches en diversité biologique et qui permettent une préservation viable dans le long terme.

Ainsi que le remarquent certains écologistes ou que le mettent en œuvre certaines fondations (le WWF par exemple), l'heure n'est plus à la protection de zones trop dégradées mais bien plutôt à une concentration maximale des moyens sur des secteurs encore peu exploités. À partir de ces secteurs suffisamment vastes et préservés, une bonne gouvernance nationale et internationale des milieux naturels a quelques chances de succès. Ces secteurs naturels sont en revanche des espaces où les populations humaines rencontrent de forts problèmes politiques, économiques et d'acculturation, ce qui explique aussi en grande partie les destructions causées. Ou à l'inverse, et c'est ce que nous promouvons dans le cadre de recherches en Côte d'Ivoire, Guinée ou Ghana, les zones non désignées (en tant que parc, réserve, sanctuaire...) où coexistent brousse et espaces civilisés, animaux et hommes, sont souvent les lieux les plus inatendus et prometteurs pour tenter de nouveaux échanges et construire de nouveaux savoirs partagés (Leblan 2006, Yamakoshi 2006). Ce sont bien évidemment non des questions de « nature », mais des questions anthropologiques, qu'il faut avoir à l'esprit et traiter sous 


\section{L’animal spectaculaire}

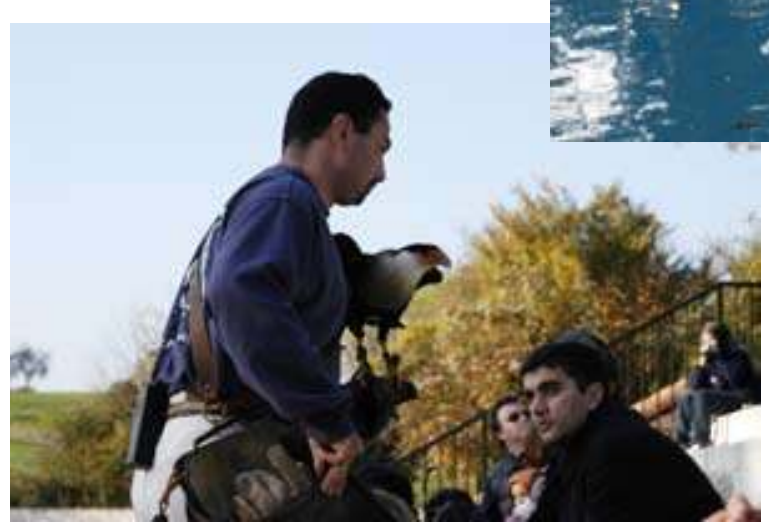

\section{Démonstrations}

Zoo Parc de Beauval, 2002.
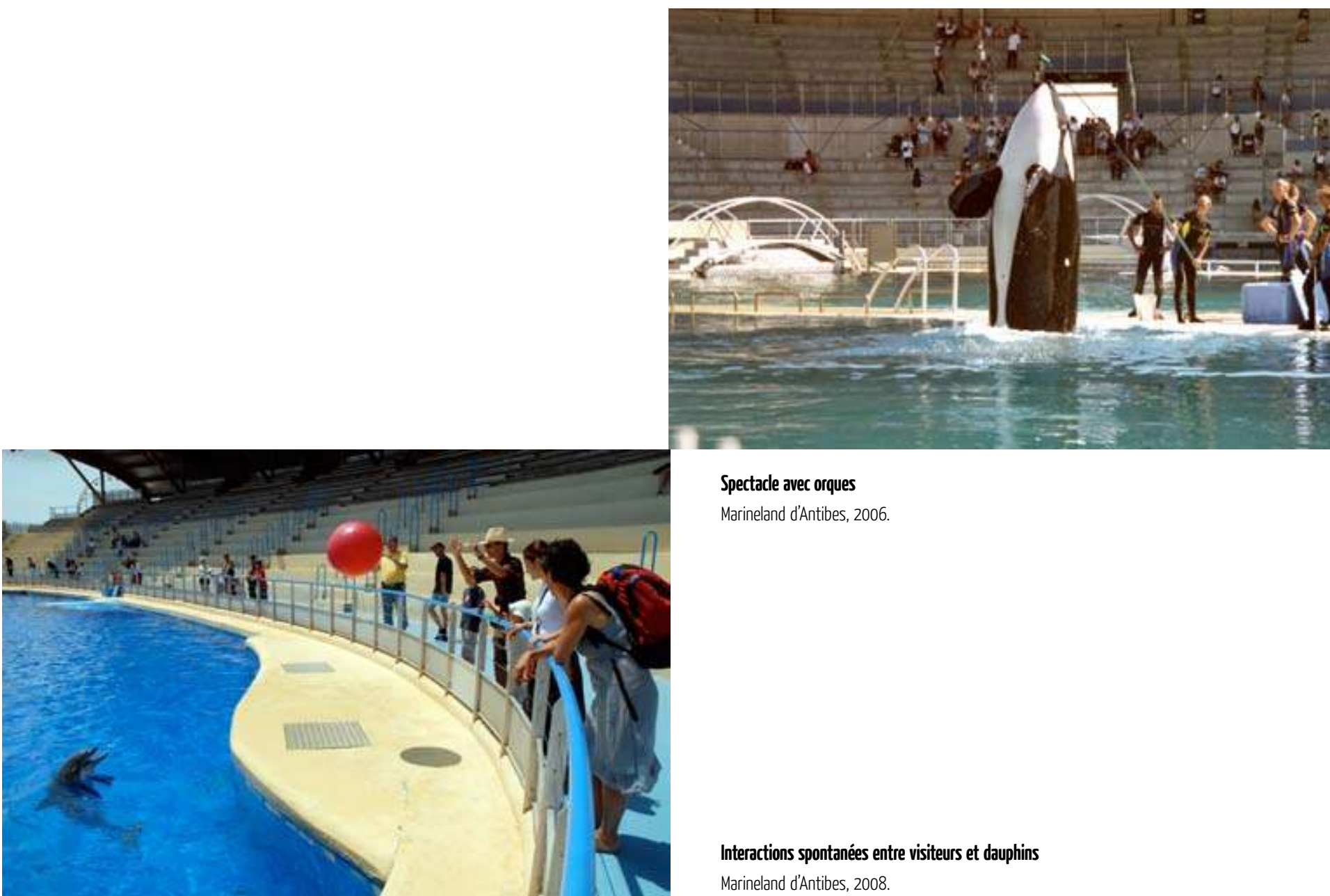

Spectacle avec orques

Marineland d'Antibes, 2006. 
peine de voir naître des conflits d'intérêts très nets entre les sociétés privilégiées du Nord et les sociétés déphasées du Sud.

Car au final, ralentir les dégradations actuelles de l'environnement et l'érosion de la diversité animale revient, selon nous, à ne pas se méprendre sur les causes des problèmes et sur les cohérences et incohérences de la science; autrement dit, il s'agit d'abord de bien identifier les causes anthropiques qui génèrent les problèmes de "nature », en tordant de nouveau le cou au dualisme «nature/culture».

Maintenant, il semble que l'objectif visant à élever des espèces rares dans des conservatoires constitués par les zoos ne peut être que secondaire au regard de l'urgence des actions de terrain. Les destructions de la nature sont générées en grande partie par les difficultés à vivre ou survivre de multiples populations. Si certains zoos estiment participer à un sauvetage de la nature par des élevages, l'action ne doit pas, selon nous, servir d'alibi à leur propre existence. D'autres, comme ceux de San Diego, Francfort, Jersey ou Mulhouse, ont depuis longtemps pris conscience d'un rôle à jouer sur le terrain en finançant des projets qui aident certains pays à renforcer les mesures de protection de zones clefs. À quel point agissent-ils efficacement, autrement dit, de façon anthropologiquement éclairée et politiquement responsable? Nous ne pouvons y répondre simplement. Une analyse critique serait nécessaire, elle impliquerait un travail à part entière, qui mobiliserait une exploration fine des forces idéologiques et financières en jeu et prendrait bien sûr la mesure de ces nouvelles économies globales de la nature.

\section{NOTES}

Toutes les photos sont de Frédéric Joulian, sauf mention contraire.

1. Borneo Orangutan Survival Foundation. 2008 http://www.orangutan.or.id/, http://www.orangutan.or.id/bos.php?cat=program\&frame=detai l\&id=358757574319
2. Nous tenons à remercier l'Ehess, le programme « Terrains, Techniques et Théories » du ministère de la Recherche et l'ambassade de France au Ghana de nous avoir soutenu financièrement et administrativement dans ces travaux de terrains hors normes. 


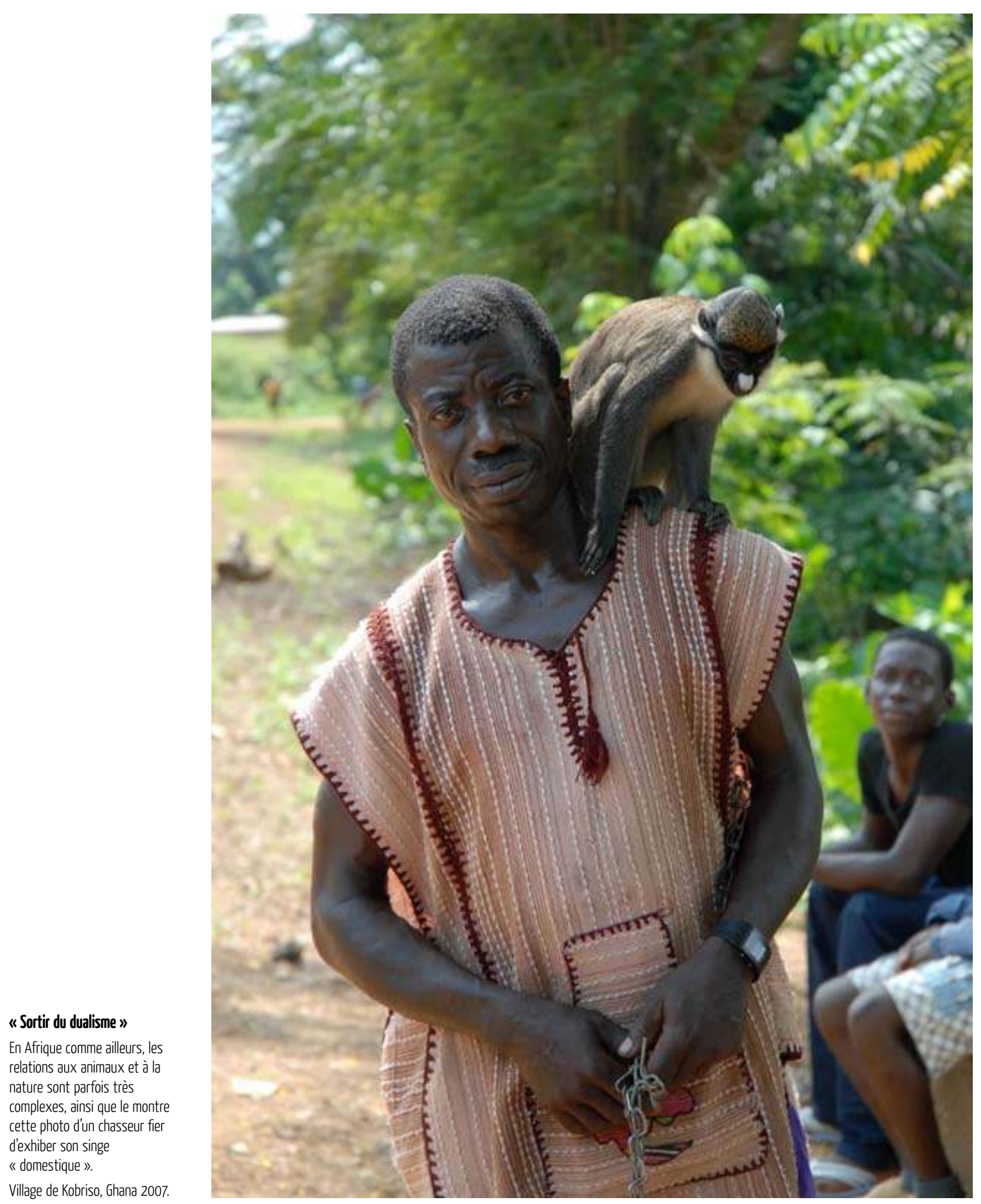




\section{RÉFÉRENCES}

Abegg, C. \& Thierry, B., 1998, «L'Lrigine des traditions chez les singes» in Ducros, A., Ducros, J. \& Joulian, F. (eds), La Culture est-elle naturelle? Histoire, épistémologie et applications récentes du concept de culture. Paris, Errance: 137-150.

André, C., 2007, Rapport annuel: « Le sanctuaire LolaYaBonobo ». Les Amis du Bonobo du Congo.

Bancel, N., 2004, Zoos humains. Au temps des exhibitions humaines. Paris, La Découverte.

Baratay, E. \& Hardouin-Fugier, E., 1998, Zoos. Histoire des jardins zoologiques en Occident (XVle-XXe siècle). Paris, La Découverte.

Beck, B. B. \& Power, M. L., 1988, «Correlates of sexual and maternal competence in captive gorillas », Zoo Biology $7:$ 339-350.

Boesch, C., \& Tomasello, M., 1998, « Chimpanzee and human cultures», Current Anthropology 49 (5): 591-614.

Brewer, S. 2008, Chimpanzee rehabilitation trust. http://www.chimprehab.com/

Brouard, P., 1994, Petite histoire des ménageries du Jardin des Plantes de Paris. Paris, SECAS.

Chouin, G., 2002, «Sacred Groves as Historical and Archaeological Markers in Southern Ghana », Institute of Development Studies Bulletin 33 (1): 39-46.

Gossens B, Ancrenaz M., Vidal C. \& Jamart A., 2001, « Captive care in Primates: applications to a chimpanzee (Pan troglodytes troglodytes) release program in the Republic of Congo », Laboratory primate Newsletter 40(4).

Jankowski, F, 2000, «Étude des méthodes de recrutement indirect sur un groupe de chimpanzés (Parc National du Banco, Côte d'Ivoire)». Mémoire de DEA, Université de Bordeaux I, (dir. F. Joulian, J-J. Hublin).

Jeangène Vilmer, J.-B., 2008, Ethique animale. Paris, PUF.

Joulian, F., 1995, « Mise en évidence de différences traditionnelles dans le cassage des noix chez les chimpanzés (Pan troglodytes) de la Côte d'Ivoire, implications paléoanthropologiques », Journal des Africanistes 65 (2): 57-77.

Joulian, F., 2002a, «Ethologie au zoo, éthologie dans la nature: le scientifique en contexte», Journée d'étude: «Élever, conserver, visiter le sauvage: des interactions entre les hommes et les animaux dans les jardins zoologiques» (dir. F. Joulian, E. Porqueres, S. de Cheveigné), Paris, Collège de FranceEHESS, 16 mai.

Joulian, F., 2002b, «Animaux, parcs et hommes en Côte d'Ivoire ». Journée d'étude: «Conservation des animaux et mise en réserve de la Nature » (dir. F. Joulian, E. Porqueres, S. de Cheveigné), Paris, Collège de France-EHESS, 13 juin.

Joulian, F., 2003, Intervention lors de la Conférence Unesco du 26-28 novembre à Paris. Expert meeting on great apes and the Great Apes Survival Project (GRASP). http://www.unesco.org/mab/grasp/E_ Fin_Rep_rev.pdf

Joulian, F., 2004, La perception de la conservation des Grands Singes dans différents contextes historiques, politiques et socioculturels. Anthropologie des relations Hommes/Grands Singes: Quelles implications pour la conservation? Congo, Patrimoine en danger « Ensuring the protection of sites in the DRC- An experts' workshop » Unesco, Paris 13/14 sept. 2004.

Joulian, F., Govoroff, N., Jankowski, F, Leblan, V., 2007, « Les traditions techniques des chimpanzés d'Afrique de l'Ouest ou comment combler le hiatus entre les populations d'Afrique de l'Ouest et d'Afrique Centrale », in 20e colloque de la SFDP « Primates: histoires d'évolutions » Session Ethologie/Cognition, Paris, Muséum National d'Histoire Naturelle, 22-23-24 octobre. Folia Primatologica, à paraître.

Karanth, K. U., 1992, « Conservation prospects for Lion-tailed macaques in Karnataka, India », Zoo Biology $11: 33-41$. 
Leblan, V., Joulian, F, 2006, « Chimpanzees (Pan troglodytes verus) in anthropic environments. Ecology and ethnohistory of their interactions with Fula and Landuma communities of the region of Kakandé (Guinea)», Folia Primatologica 78 (3): 201.

Leblan, V., 2006, «La Mise en réserve des espaces soumis aux jinna en pays peul et landouma (Guinée, préfecture de Boké). Cause d'une controverse lalente», in Selmi, Adel \& Vincent Hirtzel (eds), Gouverner la nature. Paris, LHerne: 47-64 («Cahiers d'anthropologie sociale $3 »$ ).

Leblan, V., 2007, «Analyse spatiale des relations entre les hommes et les chimpanzés dans la région de Boké (Guinée) » Thèse de doctorat, EHESS, (dir. P. Descola, F. Joulian).

Mullan, B. \& Marvin, G., 1999, Zoo Culture. Urbana \& Chicago, Univ. Illinois Press.

Muséum, 2007, Paris Meetings on primates and their habitats. 22-26 octobre, Museum National d'Histoire Naturelle, CMS, GRASP, UNEP, UNESCO, SFDP.

Oates, J., 2006, «Is the chimpanze, Pan troglodytes, an endangered species? It depends on what "endangered" means », Primates 47: 102-112.

Pétition, 2008, Manifeste pour les grands singes et la nature. http://terresacree.org/manifestegrandssinges. htm

Snowdown, C. T., 1994, "The significance of naturalistic environments for primate behavioral research », in E. F. Gibbons Jr., E. J. Wyers, E. Waters, E. W. Menzel (eds), Naturalistic environments in captivity for animal behavior research. New-York, Suny Press.

Waal, Franz de, 1982, Chimpanzee Politics. Sex and Power among Apes. NY, Haper \& Row.

Waal, Franz de, 1986 « The Brutal elimination of a rival among captive male chimpanzees », Ethology and Sociobiology $7: 237-251$.

Waal, Franz de, 1992, De la réconciliation chez les primates. Paris, Flammarion.

Wilson, E., 1989, « La diversité du vivant menacée », Pour la Science 145: 66-73.

Walsh, P.D., Abernethy, K.A.et al., 2003, « Catastrophic ape decline in western equatorial Africa », Nature 422: 611-614.

Whiten et al., 1999, «Culture in Chimpanzees », Nature 399: 682-685.

Yamakoshi, G., 2006, « An indigenous concept of landscape management for chimpanzee conservation at Bossou, Guinea », in J. Maruyama, L. Wang, T. Fujikura, M. Ito (eds), Crossing Disciplinary Boundaries and Re-visioning Area Studies: Perspectives from Asia and Africa. Proceedings of Kyoto Symposium 2006, Kyoto University, Kyoto: 3-10.

Yerkes, R. M., [1943] 1971, Chimpanzees: A laboratory colony. New York, Johnson Reprint Corporation. 


\section{RÉSUMÉ}

Zoos et cause animale. Perspectives éthologique et anthropologique. Cet article, rédigé dans un contexte de débats sur les fonctions des zoos, a été repris et actualisé au regard des avancées du terrain et des réformes des zoos. Nous interrogeons les discours des zoos sur un aspect crucial, celui de leur légitimation de l'enfermement d'animaux sauvages, et cela en examinant les différents arguments avancés: conservation d'espèces rares, recherche scientifique, éducation du public. Nous discutons également la question de la réintroduction d'animaux en milieu naturel et la pertinence de telles entreprises au regard des multiples échecs subis au fil des années. Nous interrogeons de façon critique les changements de dispositifs d'exposition (de traditionnels en cage aux « naturalistes enrichis ») qui renvoient à de nouveaux modèles de conception et d'administration de la nature. Au final, nous analysons les contradictions majeures entre causes animales et dimensions anthropologiques ou conservatoires, et plus largement, l'asymétrie politique Nord-Sud dont les questions de conservation et d'exposition d'animaux sont de pertinents indicateurs.

\section{ABSTRACT}

Zoos and the Animal Cause. Ethological and Anthropological Perspectives. This paper, written in the particular context of the debate about the functions of zoos, has been revised and actualized, including present day discussions and new fieldwork. We question the discourse of zoos on a main point, that of how they, nowadays, legitimate the shutting in of wild animals. We present the different justifications that are given : conserving rare species, scientific research, informal public education. We also discuss the reintroduction of zoo animals in natural surroundings and we evaluate the real impact of such attempts over the past decades. We question, in a critical way, the change in exhibition settings (from traditional ones to « naturalist and enriched » ones), which reflect new conceptions and ways of administrating of nature. Finally, we examine the major contradictions between the animal cause and conservationist or anthropological implications. We also consider the political asymmetry between North and South of which the conservation and exhibition of animals are very good indicators.

\section{MOTS CLÉS}

Jardin zoologique, conservation, réintroduction, nature, enfermement, dialogue Nord-Sud.

\section{KEYWORDS}

Zoological garden, conservation, reintroduction, nature, confinement, North-South dialogue. 